\title{
On Equivalence Relation in Factor Algebras
}

\author{
Yassin A. Salih \\ Department of Operations Research \\ College of Computers and Mathematics Seines \\ University Mosul
}

Received

$26 / 02 / 2012$
Accepted

06 / 06 / 2012

$$
\begin{aligned}
& \text { المؤثرات الموجبة والعلاقة "G ح" (G - لمرة) بين المساقط في جبور العوامل الموسعة. }
\end{aligned}
$$

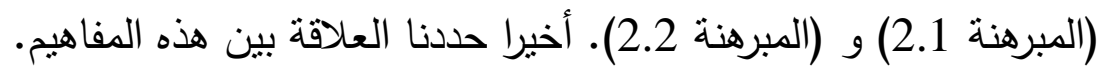

\begin{abstract}
In this paper we introduce and study a relation " " between projections, a relation " $\approx$ " on positive operators and a relation " $\sim$ G" $(G$ group) on projections in extended factor algebras. The main results in this paper state that these relations are indeed an equivalence relation (Theorem 2.1) and (Theorem 2.2). Finally, we determine the relation between these concepts.
\end{abstract}

\section{Introduction}

In this work three kinds of relations are introduced and studied. The first one [Def.1.4] between projections, the second relation [Def.1.5] applicable to all positive operators and the third relation [Def.1.6] between projections on extended factor algebra.

We refer the reader to the references [1], [2], [4], [6] and [7], as standard background references for $C^{*}$-and von Neumann algebras and for the subject.

Following [2],[6] we introduce some concepts which will be use in various places in this paper. We denote by $B(H)$ as the algebra of all bounded linear operators acting on a Hilbert space $H$ and let $A$ be a subset of $B(H)$. By $A^{\prime}$ we denote the set of all elements of $B(H)$ commuting with 
every element in $A$, then $A^{\prime}$ is called the commutant of $A$. Let $\left(A^{\prime}\right)^{\prime}=A^{\prime \prime}$, $A^{\prime \prime}$ is called the bicommutant of $A$. It is clear that $A^{\prime}$ is Banach algebra containing the identity I. If $x \in A$ implies $x^{*} \in A$, then $A^{\prime}$ is $C^{*}$-algebra acting on $H$, where $*$ is involution (or *- operator).

Recall that an algebra $A$ is said to be semiprime if for any ideal $I$ of $A$ such that $I^{2}=0$ implies that $I=0$.

\subsection{Definition: [6]}

*- subalgebra $A$ of an algebra $B(H)$ is called a von Neumann algebra on $H$ if $A=A^{\prime \prime}$. For example $B(H)$ is von Neumann algebra.

\subsection{Definition:}

Let $A$ be a semiprime von Neumann algebra, the extended centeroid of $A$ is denoted by $C(A)$ and defined by

$$
C(A)=\{f: I \rightarrow A \mid f(x a)=f(x) a, f(a x)=a f(x), \forall a \in A, x \in I\},
$$

where $I$ is anonzero ideal of $A$ and $f$ is a linear mapping.

Let $A$ be a semiprime von Neumann algebra with identity, we recall that an element $a \in A$ is self-adjoint or hermitian if $a=a^{*}$, unitary if $a^{*} a=a a^{*}=I$ when $A$ contains the identity $I$, a projection if $a^{2}=a$ and $a^{*}=a$, by $A^{+}$we denoted the positive portion of $A$, i.e. $A^{+}=\left\{a^{2}: a \in A\right\}$.

We call the extended centeroid of semiprime von Neumann algebra by an extended factor algebra.

\subsection{Definition: [6]}

Let $e$ and $f$ be two projections in extended factor algebra $F, e$ is said to be equivalent to $f$ (written $e \sim f$ ), if there exists a partial isometry $v$ in $F$ such that $e=v^{*} v$ and $f=v v^{*}$.

Observe that if $v^{*} v$ is a projection, then $v v^{*}$ is automatically also a projection.

If $e \sim e_{1} \leq f$ for some projection $e_{1} \in F$, then we write $e \lesssim f$.

\subsection{Definition: [3]}

Let $F$ be an extended factor algebra and let $s$ and $t$ in $F^{+}$, we write $s \approx t$ if there is a set of elements $\left\{a_{i}\right\}$ in $F$ such that

and we say $s$ is equivalent to $t$.

$$
s=\sum a_{i}^{*} a_{i} \quad \text { and } \quad t=\sum a_{i} a_{i}^{*}
$$

In other words, $s \approx t$ if $s$ and $t$ are the l.u.b. for the sets of finite sums of $a_{i}^{*} a_{i}$ 's and $a_{i} a_{i}^{*} ' s$ respectively.

For $s$ and $t$ in $F^{+}$, we write $s \underset{\approx}{\gtrless}$ if there is $r$ in $F^{+}$such that $s \approx r \leq t$. 
Now let $A$ be a semiprime von Neumann algebra acting on a Hilbert space $H$. Let $G$ be a group and $t \rightarrow u_{t}$ a unitary representation of $G$ on $H$ such that $u_{t}^{*} A u_{t}=A$ for all $t \in G$.

1.5. Definition: ( $G$ - equivalence) [5]

Let $F$ be an extended factor algebra. If $e$ and $f$ are projections in $F$ we say, $e$ is $G$-equivalent to $f$ (written $e \sim{ }_{G} f$ ) if for each $t \in G$ there is an operator $k_{t} \in F$ such that

$$
e=\sum_{t \in G} k_{t} k_{t}^{*} \quad \text { and } \quad f=\sum u_{t}^{*} k_{t}^{*} k_{t} u_{t} .
$$

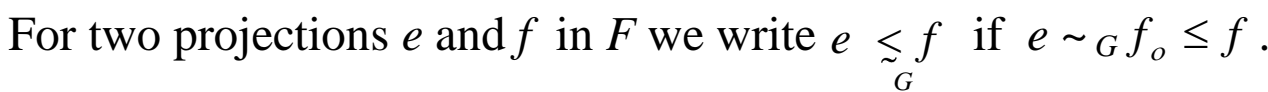

\section{The main results:}

In this section we give the main results which state that the relations " $\sim "$ " " $\approx$ " and " $\sim$ " are indeed an equivalence as shown in the following theorems. One can easily see that the relation " $\sim$ " satisfies the conditions of equivalence.

\subsection{Theorem:}

Let $F$ be an extended factor algebra. The relation $" \approx "$ is an equivalence relation in $F^{+}$.

\section{Proof:}

(i). Clearly $s \approx s$ for any $s$ in $F^{+}$.

(ii). Let $s \approx t$, then there exist $\left\{a_{i}\right\} \subset F$ such that

$$
s=\sum a_{i}^{*} a_{i} \text { and } t=\sum a_{i} a_{i}^{*} .
$$

Put $b_{i}=a_{i}^{*}$, then we get

Hence we get $t \approx s$.

$$
s=\sum b_{i} b_{i}^{*} \text { and } t=\sum b_{i}^{*} b_{i} .
$$

(iii). To prove the transitivity, let $s \approx t$ and $t \approx r$, then there exists two sets $\left\{a_{i}\right\}$ and $\left\{b_{i}\right\}$ in $F$ such that

$$
s=\sum a_{i}^{*} a_{i}, \quad t=\sum a_{i} a_{i}^{*}=\sum b_{j}^{*} b_{j}, \quad r=\sum b_{j} b_{j}^{*} .
$$

Using [3, Prop. 2.4], then there is a set $\left\{c_{i j}\right\}$ in $F$ such that

$$
\begin{aligned}
& a_{i}^{*} a_{i}=\sum_{j} c_{i j}^{*} c_{i j} \quad \text { and } \quad b_{j} b_{j}^{*}=\sum_{i} c_{i j} c_{i j}^{*} \text {. } \\
& \text { Now } s=\sum_{i} a_{i}^{*} a_{i}=\sum_{i} \sum_{j} c_{i j}^{*} c_{i j} \\
& r=\sum_{j} b_{j} b_{j}^{*}=\sum_{j} \sum_{i} c_{i j} c_{i j}^{*} .
\end{aligned}
$$

Hence we get $s \approx r$ which complete the proof. $\Delta$

In order to prove that " $\sim_{\mathrm{G}}$ " is an equivalence relation on the projections in the extended factor algebra $M$, we need the following structure. Let $A$ be a simeprime von Neumann algebra acting on a Hilbert 
space $H, G$ is a group and $t \rightarrow u_{t}$ is a unitary representation of $G$ on $H$ such that $u_{t}^{*} A u_{t}=A$ for all $t \in G$. For $t \in G$ let $H_{t}$ be a Hilbert space of the same dimension as $H$ and $J_{t}$ an isometry of $H$ onto $H_{t}$. Let $\tilde{H}=\sum_{t \in G} \oplus H_{t}$. We write an operator $r \in B(\tilde{H})$ (the bounded operators on $\tilde{H}$ ) - as a matrix $\left(r_{s, t}\right)_{s, t \in G}$, where $r_{s, t}=J_{s}^{*} r J_{t} \in B(H)$.

For each $k \in A$ let $\Phi(k)$ denoted the element in $B(\tilde{H})$ with matrix $\left(r_{s, t}\right)$ where $r_{s, t}=0$ if $s \neq t$ and $r_{s, s}=k$ for all $s \in G$. Then $\Phi$ is a *- isomorphism of $A$ onto a von Neumann subalgebra $\tilde{A}$ of $B(\tilde{H})$. For $y \in G$ let $\tilde{u}_{y}$ be the operator in $B(\tilde{H})$ with matrix $\left(r_{s, t}\right)$, where $r_{s, t}=0$ if $s t^{-1} \neq y, r_{y t, t}=u_{y}$ for all $t \in G$.

Then (see [ 1, Ch. I $\S 9$ ]) $y \rightarrow \tilde{u}_{y}$ is a unitary representation of $G$ on $\tilde{H}$ such that $\tilde{u}_{y}^{*} \Phi(k) \tilde{u}_{y}=\Phi\left(u_{y}^{*} k u_{y}\right), y \in G, k \in A$.

Let $B$ denotes the semiprime von Neumann algebra generated by $\tilde{A}$ and the $\tilde{u_{y}}, y \in G$, then each operator in $B$ is represented by a matrix $\left(r_{s, t}\right)$ where $r_{s, t}=k_{s t^{-1}} u_{s t^{-1}}, k_{s t^{-1}} \in A$. Now we write $p \sim q$ for two projections $p$ and $q$ in $B$ and we mean that they are equivalent, i.e. there is a partial isometry $v \in B$ such that $v v^{*}=p, v^{*} v=q$.

The next theorem includes the fact that $" \sim_{G}$ " is an equivalence relation and shows more, namely that $\sim_{\mathrm{G}^{-}}$equivalence is the same as equivalence " " in $B$.

\subsection{Theorem:}

Let $e$ and $f$ be projections in extended factor algebra $M$. Then $e \sim_{G} f$ if and only if $\Phi(e) \sim \Phi(f)$. Hence $\sim_{G}$ is an equivalence relation on the projections in $M$.

\section{Proof:}

Suppose $e \sim_{G} f$. Then for each $t \in G$, there is $k_{t} \in M$ such that

Then we get

$$
e=\sum_{t \in G} k_{t} k_{t}^{*} \quad \text { and } \quad f=\sum u_{t}^{*} k_{t}^{*} k_{t} u_{t} \text {. }
$$

$$
\begin{aligned}
\Phi(e)=\sum_{t} \Phi\left(k_{t} k_{t}^{*}\right) & =\sum_{t} \Phi\left(k_{t}\right) \Phi\left(k_{t}\right)^{*} \\
& =\sum_{t}\left(\Phi\left(k_{t}\right) \tilde{u}_{t}\right)\left(\Phi\left(k_{t}\right) \tilde{u}_{t}\right)^{*}
\end{aligned}
$$

and

$$
\begin{aligned}
\Phi(f)=\sum_{t} \Phi\left(u_{t}^{*} k_{t}^{*} k_{t} u_{t}\right) & =\sum_{t} \tilde{u}_{t}^{*} \Phi\left(k_{t}^{*} k_{t}\right) \tilde{u_{t}} \\
& =\sum_{t}\left(\Phi\left(k_{t}\right) \tilde{u}_{t}\right)^{*}\left(\Phi\left(k_{t}\right) \tilde{u}_{t}\right) .
\end{aligned}
$$


Thus by [3, Th. 4.1] $\Phi(e) \sim \Phi(f)$.

Conversely assume $\Phi(e) \sim \Phi(f)$. Then there is a partial isometry $v \in M$ such that

$$
v v^{*}=\Phi(e), \quad v * v=\Phi(f) .
$$

Let $v=\left(k_{s t^{-1}} u_{s t^{-1}}\right)$, then an easy calculation show

$$
e=\sum_{t \in G} k_{t} k_{t}^{*} \text { and } f=\sum u_{t}^{*} k_{t}^{*} k_{t} u_{t},
$$

hence $e \sim_{G} f$ and the proof is complete. $\Delta$

Throughout the following notes we can obtain an interesting and important relation between the equivalences.

\section{Note 1:}

If $G$ is the one element group, then " $\sim_{G}$ implies to " $\sim_{G}$ ".

\section{Note 2:}

If $G$ is the additive group and the representation $t \rightarrow u_{t}$ is the trivial representation, i.e. $u_{t}=I$ for $t \in G$, then $" \sim_{\mathrm{G}}$ implies to $" \approx "$.

\section{Note 3:}

If $e$ and $f$ are equivalent projections in extended factor algebra $F$, i.e. there is a partial isometry $v \in F$ such that $e=v v^{*}, f=v^{*} v$, then $e \sim_{G} f\left(e \sim f \Rightarrow e \sim_{G} f\right)$. Clearly form the definition of $\sim_{\mathrm{G}}$ by putting $k_{e}=v$, $k_{t}=0$ for $t \neq e$.

\section{Acknowledgements}

We would like to express our gratitude to referees for several useful suggestions concerning the results in this paper.

\section{REFERENCES}

1) Dixmier J., Les algebras d'operateurs dans l'espace hilbertien (Cahiers Scientifiques 25), Gauthier-Villars, Pairs, 1957.

2) Dixmier J., " $\mathrm{C}^{*}$-Algebras", North-Holland publishing company, Amsterdam, New York, Oxford, 1977.

3) Kadison R.V. and Pedersen G.K., Equivalence in operator algebras, Math. Scand., 27 (1970), 205-222.

4) Morrison T. J., "Functional Analysis an Introduction to Banach Space Theory", John Wiley \& Sons, Inc., 2001.

5) Stormer E., Automorphisms and equivalence in von Neumann algebras, Pacific. J. Math. Vol.44, No.1, 1973.

6) Takesaki M., "Theory of Operator Algebras I", Springer-Verlag, New York Inc., 1979.

7) Yoshino T., "Introduction to Operator Theory", John Wiley \& Sons, Inc., 1993. 